Homology, Homotopy and Applications, vol.6(1), 2004, pp.5-16

\title{
AUTOUR DES FORMES QUADRATIQUES QUASI-VOISINES
}

\author{
AHMED LAGHRIBI \\ (communicated by Ulf Rehmann)
}

\begin{abstract}
In this article we study a generalization of the notion of Pfister neighbors. An anisotropic quadratic form $\varphi$ over a field $F$ of characteristic not 2 is called a quasi-Pfister neighbor when the anisotropic part $\left(\varphi_{F(\varphi)}\right)_{\text {an }}$ is $F(\varphi)$-similar to an $F$ quadratic form $\psi$ where $F(\varphi)$ denotes the function field of the projective quadric given by $\varphi$. We prove the uniqueness of $\psi$ up to $F$-similarity for forms $\varphi$ of dimension $\leqslant 8$, odd dimension and many others of large dimension, and in these cases we give a precise description of $\psi$.
\end{abstract}

\section{Introduction}

Soit $F$ un corps de caractéristique $\neq 2$. Pour une forme quadratique $\varphi$, on note $\operatorname{dim} \varphi$ sa dimension, $\operatorname{det} \varphi$ son déterminant, $\varphi_{\text {an }}$ sa partie anisotrope, $C(\varphi)$ son algèbre de Clifford et $C_{0}(\varphi)$ son algèbre de Clifford paire.

A une forme quadratique $\varphi$ de dimension $\geqslant 2$, non isométrique au plan hyperbolique $\langle 1,-1\rangle$, on associe la quadrique projective $X_{\varphi}$ d'équation $\varphi=0$. On note $F(\varphi)$ le corps des fonctions de $X_{\varphi}$. Lorsque $\varphi \simeq\langle 1,-1\rangle$ ou $\operatorname{dim} \varphi \leqslant 1$, on pose $F(\varphi)=F$.

Pour $\varphi$ une forme quadratique telle que $\operatorname{dim} \varphi_{\text {an }} \geqslant 1$, on définit une suite $\left(F_{i}, \varphi_{i}\right)_{0 \leqslant i \leqslant \mathrm{~h}(\varphi)}$ de formes quadratiques et d'extensions de $F$, dite la tour de déploiement générique de $\varphi$, comme suit: $F_{0}=F, \varphi_{0}=\varphi_{\text {an }}$, et pour $i \geqslant 1$ on définit par induction $F_{i}=F_{i-1}\left(\varphi_{i-1}\right)$ et $\varphi_{i}=\left(\left(\varphi_{i-1}\right)_{F_{i}}\right)_{\text {an }}$. L'entier $\mathrm{h}(\varphi)$, appelé hauteur de $\varphi$, est le plus petit entier vérifiant $\operatorname{dim} \varphi_{\mathrm{h}(\varphi)} \leqslant 1$. Lorsque $\operatorname{dim} \varphi$ est paire et $\mathrm{h}(\varphi) \geqslant 1$, on sait que $\varphi_{\mathrm{h}(\varphi)-1}$ est semblable à une forme de Pfister $\pi$ qu'on appelle la forme dominante de $\varphi$. Dans ce cas, le degré de $\varphi$ est l'entier $d$ vérifiant $\operatorname{dim} \pi=2^{d}[\mathbf{1 8}]$.

Une forme quadratique $\varphi$ est dite une voisine de Pfister s'il existe une forme de Pfister $\pi$ telle que $2 \operatorname{dim} \varphi>\operatorname{dim} \pi$ et $\alpha \pi \simeq \varphi \perp \psi$ pour certains $\alpha \in F^{*}$ et $\psi$ une forme quadratique ( $\simeq$ désigne l'isométrie pour les formes quadratiques). Les formes $\pi$ et $\psi$ sont uniques à isométrie près. On appelle $\psi$ la forme complémentaire de $\varphi$.

L'auteur a été soutenu par le projet Européen HPRN-CT-2002-00287 “Algebraic K-Theory, Linear Algebraic Groups and Related Structures".

Received September 23, 2003, revised January 6, 2004; published on February 6, 2004.

2000 Mathematics Subject Classification: 11E04, 11E81.

Key words and phrases: Mots clés. Forme quadratique, corps des fonctions d'une quadrique projective, voisine de Pfister, quasi-voisine de Pfister, déploiement générique d'une forme quadratique. (C) 2004, Ahmed Laghribi. Permission to copy for private use granted. 
Pour un entier $n \geqslant 1$, on note $I^{n} F=(I F)^{n}$ où $I F$ est l'idéal fondamental de l'anneau de Witt $W(F)$ de $F$.

Pour $K / F$ une extension de corps et $A$ une $K$-algèbre simple centrale, on désigne par $[A]$ sa classe dans le groupe de Brauer de $K$.

Le but de cet article est de discuter un problème lié au déploiement d'une forme quadratique sur le corps des fonctions de sa propre quadrique projective. A notre connaissance ce problème n'a pas été traité jusqu'à présent. Il a son origine dans un travail dû à Kersten et Rehmann sur la classification des groupes algébriques linéaires excellents [17]. Rappelons qu'un groupe algébrique linéaire semisimple $G$ sur $F$ est dit excellent si pour toute extension de corps $K / F$, la partie anisotrope $\left(G \times{ }_{F} K\right)_{\text {an }}$ du groupe $G \times{ }_{F} K$ est définie sur $F$, c'est-à-dire, il existe un $F$-groupe $H$ tel que $\left(G \times_{F} K\right)_{\text {an }}$ soit isomorphe à $H \times_{F} K$. En remplaçant $G \times_{F} K$ par $\varphi_{K}$ où $\varphi$ est une forme quadratique, on obtient la définition d'excellence pour les formes quadratiques [18]. La théorie générique de Knebusch a permis de classifier complètement de telles formes quadratiques [18], [19]. Par contre, une classification complète dans le cas des groupes algébriques linéaires excellents est loin d'être achevée, sauf pour le cas du groupe orthogonal spécial où elle a été faite par Kersten et Rehmann [17]. Leur travail a dégagé une nouvelle classe de formes quadratiques $\varphi$ définies par la propriété suivante: Il existe une suite $\left(\psi_{0}, \cdots, \psi_{\mathrm{h}(\varphi)}\right)$ de $F$-formes quadratiques telle que $\varphi_{i}$ soit $F_{i}$-semblable à $\psi_{i}$ où $\left(F_{i}, \varphi_{i}\right)_{0 \leqslant i \leqslant \mathrm{~h}(\varphi)}$ est la tour de déploiement générique de $\varphi$. Ces formes quadratiques, dites quasi-excellentes, ont été classifiées plus tard par Izhboldin et Kersten en caractéristique 0 [12].

Pour motiver la définition qui va suivre rappelons un résultat dû à Knebusch et Hoffmann:

Théorème 1. ([19], [5]) Soient $\varphi, \psi$ deux formes quadratiques sur $F$ anisotropes avec $\operatorname{dim} \psi<\operatorname{dim} \varphi$. On a que $\varphi$ est une voisine de Pfister de forme complémentaire $\psi$ si et seulement si $\left(\varphi_{F(\varphi)}\right)_{\text {an }} \simeq-\psi_{F(\varphi)}$.

Définition 1. (1) Une forme quadratique anisotrope $\varphi$ est dite quasi-voisine si $\left(\varphi_{F(\varphi)}\right)_{\text {an }}$ est $F(\varphi)$-semblable à une $F$-forme quadratique.

(2) Pour $\varphi$ quasi-voisine, on note $\mathcal{S}(\varphi)$ la classe des $F$-formes quadratiques, à $F$ similitude près, qui sont $F(\varphi)$-semblables à $\left(\varphi_{F(\varphi)}\right)_{\mathrm{an}}$.

Voici quelques exemples simples de formes quasi-voisines:

Exemples 1. (1) Une forme quasi-excellente est évidemment une quasi-voisine.

(2) Une voisine de Pfister $\varphi$ de forme complémentaire $\varphi^{\prime}$ est une quasi-voisine et $\varphi^{\prime} \in \mathcal{S}(\varphi)$ comme on le voit par le théorème 1.

(3) Une forme bonne ${ }^{1}$ de hauteur 2 est quasi-voisine. En particulier, cet exemple montre qu'il existe des quasi-voisines qui ne sont pas des voisines.

La proposition suivante décrit partiellement les formes quasi-voisines de dimension au plus 8:

${ }^{1}$ C'est-à-dire une forme dont la forme dominante est définie sur $F$. 
Proposition 1. Soit $\varphi$ une forme quadratique anisotrope sur $F$ de dimension $\leqslant 8$ qui n'est pas du type suivant:

$$
\operatorname{dim} \varphi=8 \quad \text { et } \varphi \notin I^{2} F .
$$

Alors, $\varphi$ est une quasi-voisine si et seulement si elle est de l'un des types suivants qui s'excluent mutuellement:

(1) $\varphi$ est une voisine de Pfister.

(2) $\operatorname{dim} \varphi=4$ et $\operatorname{det} \varphi \notin F^{* 2}$.

(3) $\varphi$ est de dimension 6 mais pas une forme d'Albert, isotrope mais non hyperbolique sur $F(\sqrt{-\operatorname{det} \varphi})$.

(4) $\operatorname{dim} \varphi=8$ et l'indice ind $\mathrm{C}(\varphi)$ de $C(\varphi)$ est égal à 2 ou 4 . 3 .

L'exception $(*)$ évoquée dans cette proposition sera discutée dans la proposition

D'autres définitions d'une forme quasi-voisine peuvent être données. En terme de quadriques, qu'une forme quadratique anisotrope $\varphi$ soit quasi-voisine équivaut à l'existence d'une quadrique projective $X$ sur $F$ telle que la quadrique $X_{F(\varphi)}$ soit isomorphe à celle donnée par $\left(\varphi_{F(\varphi)}\right)_{\text {an }}$.

Dans le langage des groupes algébriques, on obtient par un résultat de Kersten et Rehmann qu'une forme quadratique $\varphi$ anisotrope est une quasi-voisine si et seulement si la partie anisotrope du groupe $S O(\varphi) \times_{F} F(\varphi)$ est définie sur $F[\mathbf{1 7}$, Th. 2.2 et 2.3].

Dans cet article on s'intéresse à la finitude de la classe $\mathcal{S}(\varphi)$ pour $\varphi$ une quasivoisine. Dans ce sens, on va donner des exemples non triviaux de formes quasivoisines $\varphi$ où $\mathcal{S}(\varphi)$ est finie. Cela couvre les formes de dimension $\leqslant 8$, de dimension impaire et d'autres formes de dimension grande. Dans tous nos exemples, on va montrer que la classe $\mathcal{S}(\varphi)$ est réduite à un seul élément, et dans tous les cas on va décrire de manière explicite cet élément (voir théorèmes 2 et 3 ). Cependant, il semble que la finitude de $\mathcal{S}(\varphi)$ est très difficile à étudier pour une quasi-voisine $\varphi$ quelconque, et on ne sait pas répondre à cela au moins pour des formes particulières comme les voisines de Pfister. Ceci motive le problème suivant:

Problème 1. Soit $\varphi$ une forme quadratique quasi-voisine. Est-ce que $\mathcal{S}(\varphi)$ est finie? Plus particulièrement, est-ce que $|\mathcal{S}(\varphi)|=1$ ? Sinon, pour quel type de formes quadratiques $\varphi$ a-t-on la finitude de $\mathcal{S}(\varphi)$ ?

En parlant de finitude, on commence par un résultat qui montre que dans certains cas les motifs des quadriques projectives des éléments de $\mathcal{S}(\varphi)$ sont tous isomorphes:

Proposition 2. Soit $\varphi$ une forme quadratique quasi-voisine telle que $\operatorname{dim}\left(\varphi_{F(\varphi)}\right)_{\text {an }} \leqslant 2^{n}<\operatorname{dim} \varphi$ pour un certain entier $n \geqslant 1$. Alors, pour toutes formes $\psi, \psi^{\prime} \in \mathcal{S}(\varphi)$, les quadriques projectives $X_{\psi}$ et $X_{\psi^{\prime}}$ ont des motifs de Chow isomorphes.

Enonçons maintenant nos résultats concernant le problème 1:

Théorème 2. Soit $\varphi$ une forme quadratique sur $F$ anisotrope et $\varphi_{1}=\left(\varphi_{F(\varphi)}\right)_{\text {an }}$. Soit $h$ (resp. d) la hauteur (resp. le degré) de $\varphi$. Si $\varphi$ est quasi-voisine, alors $\mathcal{S}(\varphi)$ 
est réduite à un seul élément dans les cas suivants:

(1) $\varphi$ est de dimension impaire. Dans ce cas, $\varphi$ est voisine de Pfister et $\mathcal{S}(\varphi)=\left\{\varphi^{\prime}\right\}$ où $\varphi^{\prime}$ est sa forme complémentaire.

(2) $\varphi$ est de dimension paire, bonne de hauteur 2. Dans ce cas, $\mathcal{S}(\varphi)=\{\tau\}$ où $\tau$ est la forme dominante de $\varphi$.

(3) $\varphi$ est quasi-excellente de hauteur $\geqslant 3$ avec $F$ de caractéristique 0 si $d \geqslant 2$. Dans ce cas, on a:

- Si $h>3$ ou $\operatorname{dim} \varphi_{h-2}$ n'est pas une puissance de 2 , alors $\varphi$ est une voisine de Pfister et $\mathcal{S}(\varphi)=\left\{\varphi^{\prime}\right\}$ où $\varphi^{\prime}$ est sa forme complémentaire.

- Si $h=3$ et $\operatorname{dim} \varphi_{h-2}$ est une puissance de 2 , alors $\mathcal{S}(\varphi)=\{\psi\}$ où $\psi$ est une $(d+1, d)$-forme de Pfister ${ }^{2}$.

(4) $\varphi$ n'est ni voisine de Pfister ni bonne, de hauteur 3 et de degré 2 avec $F$ de caractéristique 0 . Dans ce cas, on a nécessairement $\operatorname{dim} \varphi=8$, ind $\mathrm{C}(\varphi)=4$ et $\mathcal{S}(\varphi)=\{\psi\}$ où $\psi$ est une forme d'Albert ${ }^{3}$ vérifiant $[C(\varphi)]=[C(\psi)]$.

(5) $\varphi$ est de l'un des quatre types décrits dans la proposition 1. Dans ce cas:

- Si $\varphi$ est de type (1), alors $\mathcal{S}(\varphi)=\left\{\varphi^{\prime}\right\}$ où $\varphi^{\prime}$ est sa forme complémentaire.

- Si $\varphi$ est de type (2), alors $\mathcal{S}(\varphi)=\{\langle 1,-\operatorname{det} \varphi\rangle\}$.

- Si $\varphi$ est de type (3), alors $\mathcal{S}(\varphi)=\{\psi\}$ où $\psi$ est une forme de dimension 4 , de déterminant $-\operatorname{det} \varphi$ et vérifiant $\left[C_{0}(\psi)\right]=\left[C_{0}(\varphi)\right]$.

- Si $\varphi$ est de type (4), alors $\mathcal{S}(\varphi)=\{\psi\}$ où $\psi$ est une 2-forme de Pfister ou une forme d'Albert vérifiant $[C(\psi)]=[C(\varphi)]$ suivant que ind $\mathrm{C}(\varphi)=2$ ou 4 .

Dans les assertions (3) et (4) de ce théorème, on suppose $F$ de caractéristique 0 . Cela est dû essentiellement au fait que dans les preuves on utilise des résultats connus qu'en cette caractéristique.

En dimension 10 et 12 on a le résultat suivant:

Théorème 3. (1) Il n'existe pas de forme quasi-voisine $\varphi \in I^{2} F$ de dimension 10 telle que ind $\mathrm{C}(\varphi)=2$.

(2) Il existe des formes quasi-voisines $\varphi \in I^{2} F$ de dimension 12 telles que ind $\mathrm{C}(\varphi)=2$. Pour une telle forme $\varphi$ on a que $\mathcal{S}(\varphi)$ est réduite à un seul élément. Plus précisément, $\operatorname{dim}\left(\varphi_{F(\varphi)}\right)_{\mathrm{an}} \in\{4,8,10\}$ et dans chaque cas on a:

- Si $\operatorname{dim}\left(\varphi_{F(\varphi)}\right)_{\mathrm{an}}=4$, alors $\varphi$ est voisine de Pfister et $\mathcal{S}(\varphi)=\left\{\varphi^{\prime}\right\}$ où $\varphi^{\prime}$ est sa forme complémentaire.

- Si $\operatorname{dim}\left(\varphi_{F(\varphi)}\right)_{\mathrm{an}}=8$, alors $\psi \in \mathcal{S}(\varphi)$ si et seulement si $\varphi \perp \psi \perp \tau \perp a \tau \in I^{4} F$ pour un certain $a \in F^{*}$ où $\tau$ est la 2 -forme de Pfister vérifiant $[C(\tau)]=[C(\varphi)]$.

- Si $\operatorname{dim}\left(\varphi_{F(\varphi)}\right)_{\text {an }}=10$, alors $\psi \in \mathcal{S}(\varphi)$ si et seulement si $\varphi \perp$ b $\psi \in I^{4} F$ pour un certain $b \in F^{*}$.

Concernant les formes de type $(*)$ comme dans la proposition 1 , on prouve le résultat suivant:

${ }^{2} \mathrm{Au}$ sens de Hoffmann [6, Def. 3.4]

${ }^{3}$ C'est-à-dire $\operatorname{dim} \psi=6$ et $\operatorname{det} \psi \in-F^{* 2}$ 
Proposition 3. S'il existe une forme quasi-voisine $\varphi$ de dimension 8 n'appartenant pas à $I^{2} F$ alors:

(1) Pour toute forme $\psi \in \mathcal{S}(\varphi)$ on a:

(i) $\psi$ est une forme d'Albert virtuelle ${ }^{4}$.

(ii) Il existe $x \in F^{*}$ et $\tau$ semblable à une 2-forme de Pfister et divisible par $\langle 1,-\operatorname{det} \varphi\rangle$ tels que $\varphi \perp x \psi \perp \tau \in I^{4} F$. En particulier, $\varphi$ est isotrope sur $F(\sqrt{-\operatorname{det} \varphi})$.

(2) $\mathcal{S}(\varphi)$ est réduite à un seul élément.

On peut se demander si la conclusion de la proposition 2 est vraie sans l'hypothèse $\operatorname{dim}\left(\varphi_{F(\varphi)}\right)_{\text {an }} \leqslant 2^{n}<\operatorname{dim} \varphi$.

Pour tout entier pair $n \geqslant 8(n \neq 12)$ Izhboldin a construit un corps $F$ de caractéristique $\neq 2$ et une paire de formes quadratiques sur $F$ de même dimension $n$ qui ne sont pas semblables mais dont les quadriques projectives ont des motifs isomorphes [11]. Cela donnerait peut être une voie pour construire un éventuel exemple d'une forme quasi-voisine $\varphi$ telle que $|\mathcal{S}(\varphi)|>1$ (dans ce cas, $\varphi$ doit être de dimension au moins 10 comme on le voit par le théorème 2(5) et la proposition 3). Dans ce sens, rappelons aussi un résultat d'Izhboldin qui affirme que deux formes quadratiques de même dimension impaire dont les quadriques projectives ont des motifs isomorphes sont semblables [10]. En particulier, en dimension impaire la proposition 2 donne une réponse partielle au problème 1 , de plus on voit par le théorème 2(1) que cela est vraie sans hypothèse supplémentaire.

On vient de voir que certaines des formes $\varphi$ traitées dans les théorèmes 2 et 3 sont données par des voisines de Pfister et que la classe $\mathcal{S}(\varphi)$ est réduite à la forme complémentaire. La question suivante se pose alors:

Question 1. Soit $\varphi$ une forme voisine de Pfister anisotrope de forme complémentaire $\varphi^{\prime}$. A-t-on $\mathcal{S}(\varphi)=\left\{\varphi^{\prime}\right\}$ ?

Pour $\varphi$ voisine d'une $n$-forme de Pfister et de forme complémentaire $\varphi^{\prime}$, on a $\operatorname{dim} \varphi^{\prime} \leqslant 2^{n-1}<\operatorname{dim} \varphi$. Ainsi, par la proposition 2 et pour toute forme $\psi \in \mathcal{S}(\varphi)$, les quadriques projectives données $\operatorname{par} \varphi^{\prime}$ et $\psi$ ont des motifs isomorphes. Par [10] les formes $\varphi^{\prime}$ et $\psi$ sont semblables lorsque $\operatorname{dim} \varphi^{\prime} \leqslant 7$. Ainsi, la question 1 a une réponse positive pour une voisine de Pfister de forme complémentaire de dimension au plus 7. Aussi, par le théorème 2(1) on a une réponse positive à cette question lorsque $\varphi$ est voisine de dimension impaire.

On suppose le lecteur familier avec l'aspect algébrique des formes quadratiques. Pour plus de détails sur les notations et la terminologie inexpliquée on renvoie à $[\mathbf{2}],[\mathbf{1 8}],[\mathbf{1 9}],[\mathbf{2 3}]$. Les preuves des théorèmes 2 et 3 nécessiteront d'importants résultats sur le problème d'isotropie, la théorie de déploiement générique des formes quadratiques et le problème de descente étudié dans [14].

Remerciements. Je tiens à remercier Bruno Kahn et Ulf Rehmann pour les différentes discussions que j'ai eues avec eux concernant ce travail.

${ }^{4}$ C'est-à-dire $\operatorname{dim} \psi=6$ et $\psi$ est anisotrope $\operatorname{sur} F(\sqrt{-\operatorname{det} \psi})$ 


\section{Preuve de la proposition 1}

Soient $F_{1}=F(\varphi), \varphi_{1}=\left(\varphi_{F(\varphi)}\right)$ an et $d=\operatorname{det} \varphi$.

(1) Supposons que $\varphi$ soit quasi-voisine et soit $\psi \in \mathcal{S}(\varphi)$. Si $\varphi$ n'est pas une voisine de Pfister, alors par le théorème 2(1) $\operatorname{dim} \varphi$ est paire.

(i) $\operatorname{Si} \operatorname{dim} \varphi=4$, alors nécessairement $\operatorname{det} \varphi \notin F^{* 2}$.

(ii) $\operatorname{Si} \operatorname{dim} \varphi=6$, alors on $\operatorname{adim} \varphi_{1}=4$.

- Si $\varphi$ est une forme d'Albert, alors $\varphi$ est de hauteur 2 et donc bonne puisqu'elle est supposée quasi-voisine, ce qui n'est pas possible.

- Si $\varphi$ n'est pas une forme d'Albert, alors $\psi_{F(\sqrt{-d})}$ est semblable à une 2-forme de Pfister, et donc la forme $\varphi$ est hyperbolique sur $F_{1}(\sqrt{-d})(\psi)$. Ainsi, $\varphi$ est hyperbolique sur $F(\sqrt{-d})(\psi)$ et donc $\varphi_{F(\sqrt{-d})}$ est isotrope. Puisque $\varphi$ n'est pas une voisine de Pfister, la forme $\varphi_{F(\sqrt{-d})}$ n'est pas hyperbolique [19].

(iii) $\operatorname{Si} \operatorname{dim} \varphi=8$, alors par hypothèse $\varphi \in I^{2} F$. On a ind $\mathrm{C}(\varphi) \geqslant 2$ puisque $\varphi$ n'est pas une voisine. Puisque $\left[C(\varphi)_{F_{1}}\right]=\left[C(\psi)_{F_{1}}\right]$ et $\operatorname{dim} \varphi>4$, on obtient que $[C(\varphi)]=[C(\psi)]$ et donc ind $\mathrm{C}(\varphi)=2$ ou 4 du fait que $\psi \in I^{2} F$ de dimension $\leqslant 6$.

(2) Réciproquement, supposons que $\varphi$ soit de l'un des quatre types décrits dans la proposition.

(i) Si $\varphi$ est voisine de Pfister, alors elle est quasi-voisine.

(ii) $\operatorname{Si} \operatorname{dim} \varphi=4$ et $\operatorname{det} \varphi \notin F^{* 2}$, alors $\varphi$ est bonne de hauteur 2 et donc elle est quasi-voisine.

(iii) $\mathrm{Si} \varphi$ est de type (3): Soient $\alpha, \beta, x, y \in F^{*}$ tels que $\varphi \simeq \alpha\langle 1, d\rangle \perp$ $\beta\langle 1, x, y, x y\rangle$. Puisque $\varphi_{F(\sqrt{-d})}$ n'est pas hyperbolique, la forme $\langle d,-x,-y,-x y\rangle$ est anisotrope. Ainsi, cette forme reste anisotrope sur $F(\varphi)[\mathbf{2 4}]$. Par la multiplicativité d'une forme de Pfister, on a que $\varphi_{1}$ est semblable à $\langle d,-x,-y,-x y\rangle_{F(\varphi)}$ et donc $\varphi$ est quasi-voisine.

(iv) $\mathrm{Si} \varphi$ est de type (4): Soit $\eta$ est une 2-forme de Pfister ou une forme d'Albert vérifiant $[C(\varphi)]=[C(\eta)]$ suivant que ind $\mathrm{C}(\varphi)=2$ ou 4 . Dans ce cas on sait que $\varphi_{1}$ est semblable à $\eta_{F(\varphi)}$ et donc $\varphi$ est quasi-voisine.

\section{Preuve de la proposition 2}

Puisque $\operatorname{dim} \psi=\operatorname{dim}\left(\varphi_{F(\varphi)}\right)_{\text {an }} \leqslant 2^{n}<\operatorname{dim} \varphi$, on a que $i_{W}\left(\psi_{K(\varphi)}\right)=i_{W}\left(\psi_{K}\right)$ pour toute extension $K / F[\mathbf{5}]$. On reprend le même raisonnement pour $\psi^{\prime}$ et on utilise le fait que $\psi_{K(\varphi)}$ est semblable à $\psi_{K(\varphi)}^{\prime}$ pour avoir $i_{W}\left(\psi_{K}\right)=i_{W}\left(\psi_{K}^{\prime}\right)$. Par un résultat ${ }^{5}$ dû à Vishik [27], les quadriques projectives données par $\psi$ et $\psi^{\prime}$ ont des motifs isomorphes.

\section{Preuve du théorème 2}

Soit $\left(F_{i}, \varphi_{i}\right)_{0 \leqslant i \leqslant h}$ la tour de déploiement générique de $\varphi$. Soient $d^{\prime}=\operatorname{det} \varphi$ et $\tau$ la forme dominante de $\varphi$. On note $\sim_{F}$ la relation de $F$-similitude entre les formes

${ }^{5}$ Vishik a prouvé ce résultat en caractéristique 0, puis Karpenko l'a étendu en toute caractéristique $\neq 2[\mathbf{1 5}]$. 
quadratiques, et $\sim$ l'équivalence de Witt. Lorsque $\varphi$ est une voisine de Pfister, on désigne par $\varphi^{\prime}$ sa forme complémentaire.

Supposons que $\varphi$ soit quasi-voisine et soient $\psi \in \mathcal{S}(\varphi)$ et $\alpha \in F_{1}^{*}$ tels que $\varphi_{1} \simeq$ $\alpha\left(\psi_{F_{1}}\right)$.

(1) Supposons que $\varphi$ soit de dimension impaire: En comparant les déterminants dans la relation $\varphi_{1} \simeq \alpha\left(\psi_{F_{1}}\right)$, on peut supposer $\alpha \in F^{*}$. Ainsi, $\varphi$ est une voisine de Pfister et $\varphi^{\prime} \simeq-\alpha \psi$. Par conséquent, $\mathcal{S}(\varphi)=\left\{\varphi^{\prime}\right\}$.

(2) Supposons que $\varphi$ soit de dimension paire, bonne de hauteur 2: Puisque $\tau_{F_{1}(\psi)}$ est hyperbolique et $\operatorname{dim} \varphi>\operatorname{dim} \tau$, on obtient par le théorème de la sous-forme que $\tau \sim_{F} \psi$. Ainsi, $\mathcal{S}(\varphi)=\{\tau\}$.

(3) Supposons que $\varphi$ soit quasi-excellente de hauteur $\geqslant 3$ : Soient $\psi_{2}, \cdots, \psi_{h}$ des $F$-formes quadratiques telles que $\varphi_{i}$ soit $F_{i}$-semblable à $\psi_{i}$.

(i) Supposons que $\operatorname{dim} \varphi_{h-2}$ ne soit pas une puissance de 2: Dans ce cas, la suite $S:=\left(\varphi, \psi, \psi_{2}, \cdots, \psi_{h}\right)$ est de première espèce au sens de [12, Def. 0.3]. Par [12, Th. 0.6] $\varphi$ est une voisine de Pfister et $\varphi^{\prime} \sim_{F} \psi$. Ainsi, $\mathcal{S}(\varphi)=\left\{\varphi^{\prime}\right\}$.

(ii) Supposons que $\operatorname{dim} \varphi_{h-2}$ soit une puissance de 2: Alors, la suite $S$ est de deuxième ou troisième espèce (toujours au sens de [12]). Dans le cas de deuxième espèce, on a que $\varphi$ est une voisine de Pfister et $\varphi^{\prime} \sim_{F} \psi$ [12, Th. 0.10]. Ainsi, $\mathcal{S}(\varphi)=\left\{\varphi^{\prime}\right\}$. Dans le cas de troisième espèce, on distingue deux cas:

- Si $h>3$, alors dans ce cas $\varphi$ est une voisine de Pfister et $\varphi^{\prime} \sim_{F} \psi[\mathbf{1 2}$, Th. $0.10]$, et donc $\mathcal{S}(\varphi)=\left\{\varphi^{\prime}\right\}$.

- Si $h=3$, alors par [12, Th. 0.10] $\psi$ est semblable à une $(d+1, d)$-forme de Pfister. Pour une autre forme $\psi^{\prime} \in \mathcal{S}(\varphi)$, on a que $\psi_{F_{1}\left(\psi^{\prime}\right)}$ est isotrope et donc $\psi_{F\left(\psi^{\prime}\right)}$ est aussi isotrope du fait que $\operatorname{dim} \varphi>2^{d+1}[\mathbf{5}]$. La forme $\psi^{\prime}$ n'est pas une voisine de Pfister car sinon elle serait semblable à une $(d+1)$-forme de Pfister et donc $\varphi$ serait de hauteur 2. Puisque $\operatorname{dim} \psi^{\prime}=2^{d+1}$, on déduit par [6, Th. 5.4] que $\psi \sim_{F} \psi^{\prime}$. Ainsi, $\mathcal{S}(\varphi)=\{\psi\}$.

(4) Supposons que $\varphi$ ne soit ni voisine de Pfister ni bonne, de hauteur 3 et de degré 2: On obtient par $[\mathbf{2 2}]$ que $\operatorname{dim} \varphi=8,12,14$ ou 16.

(i) $\operatorname{Si} \operatorname{dim} \varphi=8$ : Dans ce cas $\varphi_{1}$ est une forme d'Albert et donc il en est de même pour la forme $\psi$. Puisque $\left[C(\varphi)_{F_{1}}\right]=\left[C(\psi)_{F_{1}}\right]$ et $\operatorname{dim} \varphi>4$, on déduit que $[C(\varphi)]=[C(\psi)]$ et donc ind $\mathrm{C}(\varphi)=4$. Si on reprend le même raisonnement avec une autre forme $\psi^{\prime} \in \mathcal{S}(\varphi)$, on obtient $[C(\psi)]=\left[C\left(\psi^{\prime}\right)\right]$ et par un résultat de Jacobson [13] on déduit que $\psi \sim_{F} \psi^{\prime}$.

(ii) $\operatorname{Si} \operatorname{dim} \varphi=12$ : On a $\operatorname{dim} \varphi_{1} \geqslant 6$ puisque $h \geqslant 3$.

- Si $\operatorname{dim} \varphi_{1}=6$, alors $i_{W}\left(\varphi_{F_{1}}\right)=3$ et donc une sous-forme de $\varphi$ de dimension 10 devient isotrope sur $F_{1}$, ce qui n'est pas possible par un résultat d'Izhboldin [16, Th. 7.2.1] puisque $\varphi$ n'est pas une voisine.

- Si $\operatorname{dim} \varphi_{1} \geqslant 8$ : Puisque ind $\mathrm{C}(\psi)_{\mathrm{F}_{2}}=2$, on obtient par la réduction d'indice que ind $\mathrm{C}(\psi)=2$ et donc $\varphi$ est bonne, ce qui est exclu par hypothèse.

(iii) $\operatorname{Si} \operatorname{dim} \varphi=14$ ou 16: Par un résultat d'Izhboldin [9, Th. 13.9] on sait qu'en caractéristique 0 une forme quadratique de hauteur 2 et de degré 3 qui n'est pas bonne est de dimension 12. Avec [22, Th. 6] cela implique que le cas $\operatorname{dim} \varphi=14$ n'est pas possible, et que si $\operatorname{dim} \varphi=16$ alors $\varphi$ est une $(4,2)$-forme de Pfister et donc bonne, ce qui est exclu par hypothèse. 
(5) (i) Si $\varphi$ est de type (1), alors $\operatorname{dim} \varphi^{\prime} \leqslant 3$. On reprend la même justification donnée après la question 1 pour avoir $\mathcal{S}(\varphi)=\left\{\varphi^{\prime}\right\}$.

(ii) $\mathrm{Si} \varphi$ est de type (2), alors par l'assertion (2) on a $\mathcal{S}(\varphi)=\left\{\left\langle 1,-d^{\prime}\right\rangle\right\}$ puisque dans ce cas $\varphi$ est de hauteur 2 et bonne de forme dominante $\left\langle 1,-d^{\prime}\right\rangle$.

(iii) $\mathrm{Si} \varphi$ est de type (3), alors on vient de voir dans la preuve de la proposition 1 qu'il existe $x, y, \alpha, \beta \in F^{*}$ tels que $\varphi \simeq \alpha\left\langle 1, d^{\prime}\right\rangle \perp \beta\langle 1, x, y, x y\rangle$ et $\varphi_{1}$ est semblable à $\eta:=\left\langle d^{\prime},-x,-y,-x y\right\rangle$. Puisque $\psi_{F_{1}(\eta)}$ est isotrope, la forme $\psi_{F(\eta)}$ est aussi isotrope par [24]. De nouveau la même référence implique que $\psi \sim_{F} \eta$. Ainsi, $\mathcal{S}(\varphi)=\{\eta\}$. Remarquons que la forme $\eta$ vérifie $\left[C_{0}(\eta)\right]=\left[C_{0}(\varphi)\right]$, et que toute autre forme $\eta^{\prime}$ de dimension 4 et de déterminant $-d^{\prime}$ qui vérifie $\left[C_{0}(\varphi)\right]=\left[C_{0}\left(\eta^{\prime}\right)\right]$ est semblable à $\eta$.

(iv) $\mathrm{Si} \varphi$ est de type (4): Soit $\eta$ une 2-forme de Pfister ou une forme d'Albert vérifiant $[C(\varphi)]=[C(\eta)]$ suivant que ind $\mathrm{C}(\varphi)=2$ ou 4 . Puisque $\varphi_{1}$ est semblable $\eta_{F_{1}}$, la forme $\eta_{F_{1}(\psi)}$ est isotrope. Par l'isotropie en dimension 4 et celle d'une forme d'Albert on obtient que $\psi \sim_{F} \eta$. Ainsi, $\mathcal{S}(\varphi)=\{\eta\}$.

\section{Preuve du théorème 3}

(1) Supposons qu'il existe $\varphi \in I^{2} F$ quasi-voisine de dimension 10 telle que ind $\mathrm{C}(\varphi)=2$. Soit $\psi \in \mathcal{S}(\varphi)$. Par $[7] \psi$ est de dimension 8 et divisible par une forme $\langle 1,-\alpha\rangle$ pour un certain $\alpha \in F^{*}$. Ainsi, $\varphi$ est hyperbolique sur $F(\varphi)(\sqrt{\alpha})$ et donc $\varphi_{F(\sqrt{\alpha})}$ est aussi hyperbolique. Par conséquent, $\varphi \simeq\langle 1,-\alpha\rangle \otimes \rho$ pour une certaine forme $\rho$ de dimension 5 . En comparant les déterminants on obtient $\alpha \in F^{* 2}$ et donc $\psi$ est isotrope, ce qui n'est pas possible.

(2) Pour construire des exemples de formes quasi-voisines $\varphi \in I^{2} F$ de dimension 12 telles ind $\mathrm{C}(\varphi)=2$, on peut procéder comme suit:

On prend un corps $F$ qui a une 2-forme de Pfister $\tau$ et une 3-forme de Pfister $\pi$ anisotropes, et on suppose qu'il existe $\alpha, \beta \in F^{*}$ tels que $\varphi:=\alpha \tau \perp \beta \pi$ soit anisotrope. On a bien $\varphi \in I^{2} F$ de dimension 12 et ind $\mathrm{C}(\varphi)=2$. On affirme que $\psi=(\pi \perp-\tau)_{\text {an }}$ est semblable à $\left(\varphi_{F(\varphi)}\right)_{\text {an }}$. En effet:

- Si $i_{W}(\pi \perp-\tau)=4$ ou 2 , alors $\psi$ est de dimension 4 ou 8 et donc reste anisotrope sur $F(\varphi)$.

- Si $i_{W}(\pi \perp-\tau)=1$, alors $\psi$ est de dimension 10 et reste aussi anisotrope sur $F(\varphi)$ par [16, Th. 0.0.1].

Fixons maintenant $\varphi \in I^{2} F$ quasi-voisine de dimension 12 telle que ind $\mathrm{C}(\varphi)=2$. Soient $F_{1}=F(\varphi), \varphi_{1}=\left(\varphi_{F(\varphi)}\right)_{\text {an }}$ et $\tau$ une 2-forme de Pfister telle que $[C(\varphi)]=$ $[C(\tau)]$. Prenons $\psi, \psi^{\prime} \in \mathcal{S}(\varphi)$. Puisque ind $\mathrm{C}\left(\varphi_{1}\right)=2$ on a nécessairement $\operatorname{dim} \varphi_{1} \in$ $\{4,8,10\}$.

- Si $\operatorname{dim} \varphi_{1}=4$, alors $\varphi_{1} \simeq x \tau_{F_{1}}$ pour un certain $x \in F_{1}^{*}$. Ainsi, $\varphi$ est de hauteur 2 et bonne de forme dominante $\tau$. Par le théorème 2(2) on obtient $\mathcal{S}(\varphi)=\{\tau\}$. De plus, par [14] on peut supposer $x \in F^{*}$. Ainsi, $\varphi$ est voisine de Pfister de forme complémentaire $\varphi^{\prime} \simeq-x \tau$ et donc $\mathcal{S}(\varphi)=\left\{\varphi^{\prime}\right\}$.

- Si $\operatorname{dim} \varphi_{1}=8$, alors $\psi_{F\left(\psi^{\prime}\right)}$ est isotrope puisque $\psi_{F_{1}\left(\psi^{\prime}\right)}$ est aussi isotrope. Par [20] on obtient que $\psi \sim_{F} \psi^{\prime}$. D'où l'unicité de $\psi$.

Prouvons maintenant la caractérisation de $\psi$. Puisque $\psi_{F(\tau)}$ est semblable à 
une 3-forme de Pfister $(\operatorname{car}[C(\psi)]=[C(\tau)])$, la forme $\varphi$ devient hyperbolique sur $F(\varphi)(\psi)(\tau)$. Ainsi, $\varphi$ est hyperbolique sur $F(\psi)(\tau)$. Puisque $\varphi \perp \tau \in I^{3} F$, on obtient $e^{3}(\varphi \perp \tau) \in \operatorname{Ker}\left(H^{3} F \longrightarrow H^{3} F(\tau)(\psi)\right)\left(e^{n}\right.$ et $H^{n} F$ sont respectivement le $n$-ième invariant d'Arason [1] et le $n$-ième groupe de cohomologie galoisienne modulo 2). Par $[\mathbf{1}] e^{3}(\varphi \perp \tau)_{F(\tau)}=e^{3}\left(\psi_{F(\tau)}\right)$ et donc $e^{3}(\varphi \perp \tau)_{F(\tau)}=e^{3}(\psi \perp-\tau)_{F(\tau)}$. De nouveau [1] implique qu'il existe $a \in F^{*}$ tel que $e^{3}(\varphi \perp \tau)+e^{3}(\psi \perp-\tau)=e^{3}(\tau \perp a \tau)$. Par $[25],[26]$

$$
\varphi \perp \psi \perp \tau \perp a \tau \in I^{4} F .
$$

Réciproquement, soit $\psi$ une forme de dimension 8 qui vérifie (1). Puisque $\psi \in I^{2} F$ et $[C(\psi)]=[C(\tau)]$, on sait que $\left(\psi_{F(\psi)}\right)_{\text {an }} \simeq b \tau_{F(\psi)}$ pour un certain $b \in F(\psi)^{*}$. On étend (1) au corps $F_{1}(\psi)$ pour avoir

$$
\left(\varphi_{1} \perp-a b \tau\right)_{F_{1}(\psi)} \in I^{4} F_{1}(\psi) .
$$

Par le Hautpsatz $\varphi_{1}$ est isotrope sur $F_{1}(\psi)$ et par [20] on obtient que $\varphi_{1} \sim_{F_{1}} \psi_{F_{1}}$, c'est-à-dire, $\psi \in \mathcal{S}(\varphi)$.

- Si $\operatorname{dim} \varphi_{1}=10$ : Puisque $\psi_{F_{1}\left(\psi^{\prime}\right)}$ est isotrope, on déduit par [16, Th. 0.0.1] que $\psi_{F\left(\psi^{\prime}\right)}$ est isotrope. De nouveau la même référence implique que $\psi \sim_{F} \psi^{\prime}$. D'où l'unicité de $\psi$.

Prouvons maintenant la caractérisation de $\psi$. Par [7] il existe $\pi=\langle 1\rangle \perp \pi^{\prime}$ une 3-forme de Pfister et $y \in F^{*}$ tels que $\psi \simeq y\left(\pi^{\prime} \perp-\tau^{\prime}\right)$. Soit $\alpha \in F_{1}^{*}$ tel que $\varphi_{1} \simeq \alpha(\psi)_{F_{1}}$. De cette relation on obtient

$$
(\varphi \perp \pi)_{F_{1}} \perp \alpha y \tau \in I^{4} F_{1} .
$$

Par [14] on peut supposer le scalaire $z:=\alpha y$ dans $F^{*}$. Ainsi, $e^{3}(\varphi \perp \pi \perp z \tau)_{F_{1}}=0$. Puisque $\operatorname{dim} \varphi>8$, on obtient par $[\mathbf{1}]$

$$
\varphi \perp \pi \perp z \tau \in I^{4} F .
$$

Dans (3) on change $\pi$ par $-z \pi$ pour avoir

$$
\varphi \perp b \psi \in I^{4} F
$$

où $b=-y z$.

Réciproquement, soit $\psi$ une forme de dimension 10 qui vérifie (4). Par [8] il existe $\rho$ une forme semblable à une 4 -forme de Pfister telle que $\varphi \sim-b \psi \perp \rho$. Ainsi, $\left(\varphi_{1}\right)_{F_{1}(\rho)} \sim(-b \psi)_{F_{1}(\rho)}$ et donc $\varphi_{1}$ est isotrope sur $F_{1}(\psi)(\rho)$. On applique [16, Th. 0.0.1] pour déduire que $\varphi_{1} \sim_{F_{1}} \psi_{F_{1}}$, c'est-à-dire, $\psi \in \mathcal{S}(\varphi)$.

\section{Preuve de la proposition 3}

Soient $F_{1}=F(\varphi), \varphi_{1}=\left(\varphi_{F(\varphi)}\right)$ an et $d=\operatorname{det} \varphi$. Notons $c(\varphi)$ l'invariant de Clifford de $\varphi$.

(1) Prenons $\psi \in \mathcal{S}(\varphi)$ et $\alpha \in F_{1}^{*}$ tels que $\varphi_{1} \simeq \alpha\left(\psi_{F_{1}}\right)$.

(i) Puisque $\varphi$ n'est pas une voisine, on déduit par $[\mathbf{1 4}]$ que $\operatorname{dim} \varphi_{1} \geqslant 4$.

- Montrons que $\operatorname{dim} \psi \neq 4$. Supposons le contraire et posons $\psi=$ $\beta\langle d,-x,-y, x y\rangle$. Sans perdre de généralité, on peut supposer $\beta=1$. On prend 
l'invariant de Clifford dans la relation $\varphi_{1} \simeq \alpha \psi_{F_{1}}$ pour avoir

$$
c(\varphi)_{F_{1}}=(d,-\alpha)+(x, y)_{F_{1}}
$$

On étend $(5)$ au corps $F_{1}(\sqrt{d})$ pour avoir $c(\varphi)_{F_{1}(\sqrt{d})}=(x, y)_{F_{1}(\sqrt{d})}$. Puisque $\operatorname{dim} \varphi>$ 4 , on a $c(\varphi)_{F(\sqrt{d})}=(x, y)_{F(\sqrt{d})}$. Ainsi, il existe $z \in F$ tel que

$$
c(\varphi)=(d, z)+(x, y) .
$$

On combine (5) avec (6) pour avoir $-\alpha\langle 1,-d\rangle \simeq z\langle 1,-d\rangle_{F_{1}}$. Avec la relation $\varphi_{1} \simeq \alpha\left(\psi_{F_{1}}\right)$ on obtient

$$
(\varphi \perp-z\langle 1,-d\rangle)_{F_{1}} \sim \alpha\langle 1,-x,-y, x y\rangle .
$$

Par [14, Th. 6] la forme $\alpha\langle 1,-x,-y, x y\rangle$ est définie sur $F$, disons par une forme $\tau$. Puisque $\varphi_{F_{1}} \sim(z\langle 1,-d\rangle \perp \tau)_{F_{1}}$ et que $\operatorname{dim} \varphi_{1}=4$, la forme $z\langle 1,-d\rangle \perp \tau$ est isotrope sur $F_{1}$. Par [4] la forme $z\langle 1,-d\rangle \perp \tau$ est isotrope, ce qui implique que $\varphi_{1}$ est définie sur $F$ et donc $\varphi$ est une voisine de Pfister, une contradiction.

- Supposons que $\psi$ soit de dimension 6 et isotrope sur $F(\sqrt{-\operatorname{det} \psi})=F(\sqrt{d})$. Posons $\psi=u\langle 1,-d\rangle \perp \psi^{\prime}$ pour $u \in F^{*}$ et $\psi^{\prime}$ semblable à une 2-forme de Pfister. On prend l'invariant de Clifford dans la relation $\varphi_{1} \simeq \alpha \psi_{F_{1}}$ pour avoir

$$
c(\varphi)_{F_{1}}=(u \alpha, d)+c\left(\psi^{\prime}\right)_{F_{1}} .
$$

Les mêmes techniques qu'auparavant montrent que $u \alpha\langle 1,-d\rangle \simeq(v\langle 1,-d\rangle)_{F_{1}}$ pour un certain $v \in F^{*}$. De la relation $\varphi_{1} \simeq u \alpha\langle 1,-d\rangle \perp \alpha \psi^{\prime}$, on déduit que $(\varphi \perp$ $-v\langle 1,-d\rangle)_{F_{1}} \sim \alpha \psi^{\prime}$. Par [14, Th. 6] la forme $\alpha \psi^{\prime}$ est définie sur $F$, disons par une forme $\mu$. Ainsi, $\varphi_{F_{1}} \sim(v\langle 1,-d\rangle \perp \mu)_{F_{1}}$ et donc $\varphi_{1}$ est définie sur $F$, ce qui implique que $\varphi$ est une voisine de Pfister, une contradiction.

(ii) A scalaire près, on peut supposer $\psi=\langle k, l, k l, m, n,-d m n\rangle$. On montre comme dans (i) que $\alpha\langle 1,-d\rangle \simeq(w\langle 1,-d\rangle)_{F_{1}}$ pour un certain $w \in F^{*}$. La relation $\varphi_{1} \simeq \alpha\left(\psi_{F_{1}}\right)$ implique

$$
\varphi_{F_{1}} \sim m n \alpha\langle 1,-d\rangle \perp \alpha\langle k, l, k l, m, n,-m n\rangle .
$$

Ainsi,

$$
(\varphi \perp r\langle 1,-d\rangle)_{F_{1}} \sim \alpha\langle k, l, k l, m, n,-m n\rangle
$$

avec $r=-m n w$. On obtient par [14, Th. 2] que la forme d'Albert $\alpha\langle k, l, k l, m, n,-m n\rangle$ est définie sur $F$, disons par une forme d'Albert $\nu$. Puisque les formes $\nu_{F_{1}}$ et $\alpha\langle k, l, k l, m, n,-m n\rangle$ ont la même algèbre de Clifford, on obtient par la réduction d'indice et un théorème de Jacobson [13] que $\nu \simeq-x\langle k, l, k l, m, n,-m n\rangle$ pour un certain $x \in F^{*}$. Ainsi,

$$
(\varphi \perp r\langle 1,-d\rangle \perp x\langle k, l, k l, m, n,-m n\rangle)_{F_{1}} \sim 0 .
$$

Par un résultat de Fitzgerald $[\mathbf{3}]$ on a

$$
\varphi \perp r\langle 1,-d\rangle \perp x\langle k, l, k l, m, n,-m n\rangle \in I^{4} F .
$$

Mais cette dernière relation n'est autre que

$$
\varphi \perp x \psi \perp \tau \in I^{4} F
$$


où $\tau=r\langle 1,-d, x w,-d x w\rangle$ qui est bien semblable à une 2-forme de Pfister divisible $\operatorname{par}\langle 1,-d\rangle$.

(2) Soit $\psi^{\prime \prime}$ une autre forme de $\mathcal{S}(\varphi)$. Puisque $\psi_{F_{1}(\psi)}^{\prime \prime}$ est isotrope, on déduit par [21] que $\psi_{F(\psi)}^{\prime \prime}$ est isotrope. De nouveau la même référence implique que $\psi \sim_{F} \psi^{\prime \prime}$. D'où, $\mathcal{S}(\varphi)$ est réduite à un seul élément.

\section{References}

[1] J. Kr. Arason, Cohomologische Invarianten quadratischer Formen, J. Alg. 36 (1975), 448-491.

[2] A. Borel, Linear Algebraic Groups, Seconde édition, Graduate Texts in Math., Vol. 126, Springer-Verlag, New York/Berlin/Heidelberg, 1991.

[3] R. W. Fitzgerald, Witt kernels of function field extensions, Pacific J. Math. 109 (1983), 89-106.

[4] D. W. Hoffmann, On 6-dimensional quadratic forms isotropic over the function field of a quadric, Comm. Algebra 22 (1994), 1999-2014.

[5] D. W. Hoffmann, Isotropy of quadratic forms over the function field of a quadric, Math. Z. 220 (1995), 461-476.

[6] D. W. Hoffmann, Twisted Pfister forms, Doc. Math. J. DMV 1 (1996), 67102.

[7] D. W. Hoffmann, Splitting patterns and invariants of quadratic forms, Math. Nachr. 190 (1998), 149-168.

[8] D. W. Hoffmann, On the dimensions of anisotropic quadratic forms in $I^{4}$, Invent. Math. 131 (1998), 185-198.

[9] O. T. Izhboldin, Fields of u-invariant 9, Ann. Math. 154 (2001), 529-587.

[10] O. T. Izhboldin, Motivic equivalence of quadratic forms, Doc. Math. J. DMV 3 (1998), 341-351.

[11] O. T. Izhboldin, Motivic equivalence of quadratic forms, II, Manuscripta Math. 102 (2000), 41-52.

[12] O. T. Izhboldin, I. Kersten, Excellent special orthogonal groups, Documenta Math. 6 (2001), 385-412.

[13] N. Jacobson, Some applications of Jordan norms to involutorial simple associative algebras, Adv. in Math. 48 (1983), 149- 165.

[14] B. Kahn, A descent problem for quadratic forms, Duke Math. J. 80 (1995), 139-155.

[15] N. A. Karpenko, Criteria for motivic equivalence for quadratic forms and central simple algebras, Math. Ann. 317 (2000), 585-611.

[16] N. A. Karpenko, Izhboldin's results on stably birational equivalence of quadrics, prépublication no. 96 sur le serveur http://www.mathematik.unibielefeld.de/LAG

[17] I. Kersten, U. Rehmann, Excellent Algebraic Groups, I, J. Algebra 200 (1998), 334-346. 
[18] M. Knebusch, Generic splitting of quadratic forms I, Proc. London Math. Soc. 33 (1976), 65-93.

[19] M. Knebusch, Generic splitting of quadratic forms II, Proc. London Math. Soc. 34 (1977), 1-31.

[20] A. Laghribi, Isotropie de certaines formes quadratiques de dimension 7 et 8 sur le corps des fonctions d'une quadrique, Duke Math. J. 85 (1996), 397-410.

[21] A. Laghribi, Formes quadratiques de dimension 6, Math. Nach. 204 (1999), $125-135$.

[22] A. Laghribi, Sur les formes quadratiques de hauteur 3 et de degré au plus 2, Doc. Math. J. DMV 4 (1999), 203-217.

[23] T. Y. Lam, The algebraic theory of quadratic forms, Seconde édition, Benjamin, New York, 1980.

[24] D. Leep, Function fields results, notes manuscrites prises par T. Y. Lam (1989).

[25] A. S. Merkurjev, A. A. Suslin, The norm residue homomorphism of degree 3, (in Russian), Izv. Akad. Nauk. SSSR Ser. mat. 54 (1990), 339-356. English translation: Math. USSR-Izv. 36 (1991), 349-368.

[26] M. Rost, Hilbert 90 for $K_{3}$ for degree-two extensions, prépublication, 1986.

[27] A. Vishik, Integral motives of quadrics, prépublication 13, MPI 1998.

This article may be accessed via WWW at http://www.rmi.acnet.ge/hha/ or by anonymous ftp at

ftp://ftp.rmi.acnet.ge/pub/hha/volumes/2004/n1a2/v6n1a2.(dvi,ps,pdf)

Ahmed Laghribi

laghribi@Mathematik.Uni-Bielefeld.DE

Universität Bielefeld

Fakultät für Mathematik

Postfach 100131

D-33501 Bielefeld

Germany 